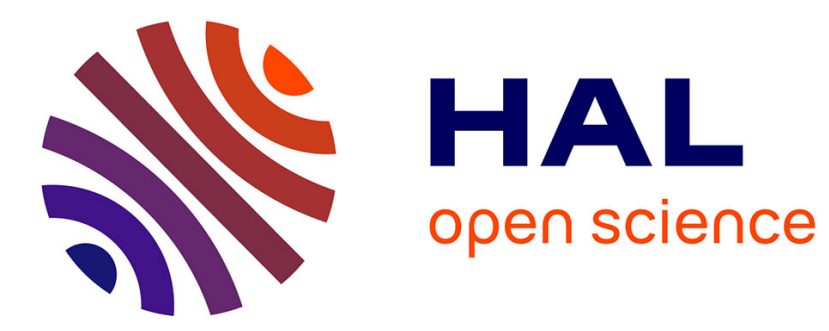

\title{
Impact of swirl and bluff-body on the transfer function of premixed flames
}

M. Gatti, R. Gaudron, Clement Mirat, Laurent Zimmer, Thierry Schuller

\section{To cite this version:}

M. Gatti, R. Gaudron, Clement Mirat, Laurent Zimmer, Thierry Schuller. Impact of swirl and bluffbody on the transfer function of premixed flames. Proceedings of the Combustion Institute, 2018, 10.1016/j.proci.2018.06.148 . hal-01877590

\section{HAL Id: hal-01877590 \\ https://hal.science/hal-01877590}

Submitted on 20 Sep 2018

HAL is a multi-disciplinary open access archive for the deposit and dissemination of scientific research documents, whether they are published or not. The documents may come from teaching and research institutions in France or abroad, or from public or private research centers.
L'archive ouverte pluridisciplinaire HAL, est destinée au dépôt et à la diffusion de documents scientifiques de niveau recherche, publiés ou non, émanant des établissements d'enseignement et de recherche français ou étrangers, des laboratoires publics ou privés. 


\title{
Impact of swirl and bluff-body on the transfer function of premixed flames
}

\author{
M. Gatti ${ }^{\mathrm{a}, *}$, R. Gaudron ${ }^{\mathrm{a}}$, C. Mirat ${ }^{\mathrm{a}}$, L. Zimmer ${ }^{\mathrm{a}}$, T. Schuller ${ }^{\mathrm{a}, \mathrm{b}}$ \\ ${ }^{a}$ Laboratoire EM2C, CNRS, CentraleSupélec, Université Paris-Saclay, 3, rue Joliot Curie, 91192 Gif-sur-Yvette cedex, France \\ ${ }^{b}$ Institut de Mécanique des Fluides de Toulouse, IMFT, Université de Toulouse, CNRS, Toulouse, France
}

\begin{abstract}
The frequency response of three lean methane/air flames submitted to flowrate perturbations is analyzed for flames featuring the same equivalence ratio and thermal power, but a different stabilization mechanism. The first flame is stabilized by a central bluff body without swirl, the second one by the same bluff body with the addition of swirl and the last one only by swirl without central insert. In the two last cases, the swirl level is roughly the same. These three flames feature different shapes and heat release distributions, but their Flame Transfer Function (FTF) feature about the same phase lag at low frequencies. The gain of the FTF also shows the same behavior for the flame stabilized by the central insert without swirl and the one fully aerodynamically stabilized by swirl. Shedding of vortical structures from the injector nozzle that grow and rollup the flame tip controls the FTF of these flames. The flame stabilized by the swirler-plus-bluff-body system features a peculiar response with a large drop of the FTF gain around a frequency at which large swirl number oscillations are observed. Velocity measurements in cold flow conditions reveal a strong reduction of the size of the vortical structures shed from the injector lip at this frequency. The flame stabilized aerodynamically only by swirl and the one stabilized by the bluff body without swirl don't exhibit any FTF gain drop at low frequencies. In the former case, large swirl number oscillations are still identified, but large vortical structures shed from the nozzle also persist at the same forcing frequency in the cold flow response. These different flame responses are found to be related to the dynamics of the internal recirculation region, which response strongly differs depending upon the mechanism adopted to stabilize the flame.
\end{abstract}

Keywords: Flame dynamics, Transfer function, Swirl number fluctuations

\footnotetext{
${ }^{*}$ Corresponding author:

Email address: marco.gatti@centralesupelec.fr (M. Gatti)
} 


\section{Introduction}

The frequency response of premixed swirling flames submitted to flow rate modulations is a topic of high scientific and technical interest due to the problems raised by combustion instabilities in gas turbines [1-3]. This response is often characterized by a Flame Transfer Function (FTF) or more recently by a Flame Describing Function (FDF) when the level of flow disturbances is considered [4].

Changing the shape of the FTF/FDF by modifying the injector design is a way to augment the stability margins of a combustor. However, there is still no systematic way to make these changes, because the dynamics of swirling flames is not fully understood $[4,5]$. Improved combustor stability is thus gained by a costly trial and error iterative process and there is a need for better knowledge of the fundamental mechanisms controlling the shape of the FTF of swirling flames.

The FTF of premixed swirling flames can be determined analytically in simplified configurations [6-8] or by numerical flow simulations in more complex geometries [9-11]. Most often this response is determined experimentally by using well proven optical techniques [12-15] even in engine like conditions [16].

Since shear layers are highly responsive to acoustic forcing, the FTF of premixed flames stabilized by a bluff body is mainly controlled by the shedding of large coherent structures, which are then convected by the mean flow and roll-up the flame. This flame roll-up process around a coherent vortical structure constitutes the main contribution controlling the FTF phase lag of premixed laminar [17] and turbulent non-swirling jet flames [18]. It also constitutes one of the fundamental process controlling the dynamics of premixed swirling flames [19-21].

It has been demonstrated that the response of the swirling vane needs to be taken into account in the dynamics of swirling flames [20, 22, 23]. Vortical transverse perturbations triggered by the axial flow disturbances at the swirler outlet lead to oscillations of the swirl level at the burner outlet. This in turn leads to oscillations of the flame angle at the anchoring point location [19]. This swirl oscillation mechanism and its impact on the FTF have been identified in several setups in which the flame is stabilized by a central bluff body $[20,22,23]$. The same dynamics is observed when the acoustic pulsation is introduced from the upstream or downstream side of the swirler [24].

In high power systems, the flame is most often fully aerodynamically stabilized without the help of any solid central insert. Giuliani et al. [25] also report large swirl number oscillations in the response of an aeronautical injector powered by kerosene when it is submitted to flow rate modulations. They however provide no FTF data. Biagioli et al. [10] analyzed the FTF of aerodynamically swirl-stabilized flames and found that the position of the Internal Recirculation Zone (IRZ) and the flame leading edge respond to the acoustic forcing by a large axial motion, but the tangential flow component is not considered in their analysis and one cannot conclude about the role of swirl oscillations.

There is yet no detailed investigation on the impact of swirl number oscillations on the FTF of swirling flames aerodynamically stabilized away from all solid components. This response is analyzed here for flames stabilized either only by a bluff body, only by swirl or by both swirl and bluff body. The premixed flames investigated feature the same equivalence ratio and the same thermal power.

The experimental setup and diagnostics are presented in section 2, followed by a description in section 3 of their flame structure in the absence of forcing. Their frequency response is analyzed in section 4 for the different injectors tested. The flow and flame dynamics at selected frequencies are investigated in section 5 to infer the swirl number fluctuations and the mechanisms controlling the response of these flames. Conclusions are finally drawn in section 6 .

\section{Experimental setup}

The experimental setup is sketched in Fig. 1. The burner is powered by a methane/air mixture. Experiments are conducted at a fixed equivalence ratio $\phi=$ 0.82 and bulk velocity $U_{b}=5.44 \mathrm{~m} / \mathrm{s}\left(T=20^{\circ} \mathrm{C}\right.$ and $p=1 \mathrm{~atm})$ in the $D=22 \mathrm{~mm}$ diameter section before the swirler unit. These conditions correspond to a constant thermal power $\mathcal{P}=5.44 \mathrm{~kW}$ assuming total combustion.

Two different radial swirlers can be fixed in the injection unit. They both feature six radial injection channels of $d_{c}=6 \mathrm{~mm}$ diameter. The design of swirlers $S_{0}$ and $S_{2}$ only differ by the distance $x$ indicated in Fig. 1 . In the first device, designated as $S_{0}$, the channels are aligned with the radial direction $(x=0 \mathrm{~mm})$. In the second one, designated as $S_{2}$, the channels are shifted from the radial direction by $x=6 \mathrm{~mm}$, to impart a strong rotation to the flow.

The flow leaves the swirler through an injector that can take two different designs. It is a straight tube of diameter $D=22 \mathrm{~mm}$ with a central rod of diameter $d=6 \mathrm{~mm}$, topped by a cone of diameter $C=14 \mathrm{~mm}$ and $10 \mathrm{~mm}$ length, to stabilize flames $S_{0}$-bb and $S_{2}$-bb 
shown in Fig. 1. The cone protrudes $2.5 \mathrm{~mm}$ inside the combustion chamber from the injector backplane. The distance between the swirler back-plane and the chamber back-plane is $L=56 \mathrm{~mm}$. For flame $S_{2}$-as at the bottom in Fig. 1, the central rod is removed and the flame is fully stabilized aerodynamically. The central injection tube is in this case slightly modified and comprises a tube with diameter $D=22 \mathrm{~mm}$ over a first section of length $L_{1}=22 \mathrm{~mm}$, followed by a nozzle of length $L_{2}=34 \mathrm{~mm}$, which is terminated by a diverging cup with an angle $\beta=15^{\circ}$. The nozzle throat diameter is in this case $D_{0}=12 \mathrm{~mm}$ in Fig. 1 .

The combustion chamber has an $82 \mathrm{~mm}$ square crosssection and a length of $150 \mathrm{~mm}$, and is equipped with four quartz windows. At the base of the burner, a loudspeaker (Monacor SP-6/108PRO, 100 Watts RMS) is mounted to pulsate the flow. The velocity is measured with a hot wire anemometer probe (Dantec Dynamics Probe 55P16 with a mini-CTA 54T30) below the swirler unit where the velocity has a top hat profile. A photomultiplier (Hamamatsu, H5784-04), equipped with a narrowband filter (Asahi Spectra, ZBPA310) centered around $310 \mathrm{~nm}$ and with a $10 \mathrm{~nm}$ bandwidth, is used to record the $\mathrm{OH}^{*}$ chemiluminescence signal.

A 2D-2C Particle Image Velocimetry (PIV) system is also used to analyze the flow structure at the injector outlet under cold flow operation. Small oil droplets of diameter $1-3 \mu \mathrm{m}$ are, in this case, seeded in the flow. The PIV system consists of $2 \times 400 \mathrm{~mJ} \mathrm{Nd}$ :YAG laser doubled at $532 \mathrm{~nm}$ operated at $10 \mathrm{~Hz}$ and a $2048 \times 2048$ $\mathrm{px}^{2}$ CCD camera (Dantec Dynamics, FlowSense EO 4M). Two different optical setups are used for longitudinal and transverse measurements, with a time delay between the two laser pulses $\Delta t=10 \mu \mathrm{s}$ and a pixel pitch of $27.9 \mathrm{px} / \mathrm{mm}$ in the first case and $\Delta t=25 \mu \mathrm{s}$ with a pixel pitch of $40.1 \mathrm{px} / \mathrm{mm}$ in the second one. Eight hundred images are taken to obtain converged mean and rms values of the velocity field, which is deduced from the cross-correlation of the PIV images by a three passes window deformation technique (from $64 \times 64 \mathrm{px}^{2}$ to $16 \times 16 \mathrm{px}^{2}$ interrogation areas), with an uncertainty of $0.1 \mathrm{px}$ on the calculated displacement.

An intensified CCD camera (Princeton Instruments, PI-MAX 4, 1024×1024 $\mathrm{px}^{2}$ ), mounted with an UV objective (Nikkor $105 \mathrm{~mm} \mathrm{f/4.5)} \mathrm{and} \mathrm{equipped} \mathrm{with} \mathrm{the}$ same filter as the photomultiplier, is also used to analyze the flame structure under steady and forced conditions. Phased averaged images of the $\mathrm{OH}^{*}$ signals and the PIV fields are synchronized by the signal driving the loudspeaker.

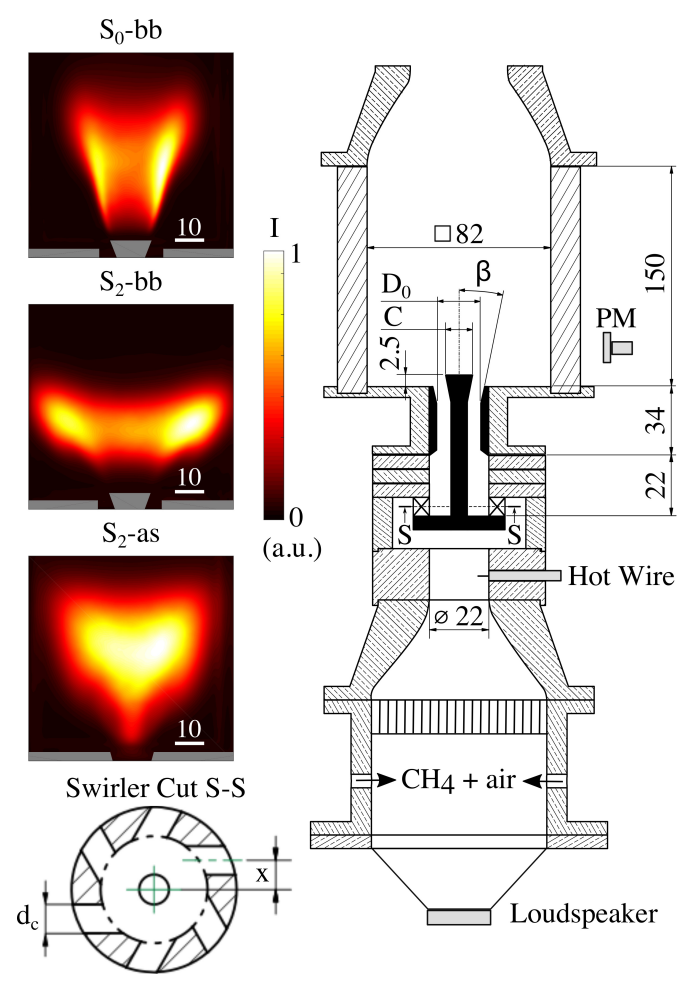

Fig. 1. Experimental setup. $S_{0}$-bb : Non-swirling flame anchored by a bluff-body, $D_{0}=22 \mathrm{~mm}, C=14 \mathrm{~mm}$. $S_{2}-b b$ : Flame stabilized by swirl $S=0.8$ and the bluff-body, $D_{0}=$ $22 \mathrm{~mm}, C=14 \mathrm{~mm}$. $S_{2}$-as : Swirling flame $S=0.75$ stabilized aerodynamically, $D_{0}=12 \mathrm{~mm}, \beta=15^{\circ}$. The main dimensions are indicated in millimeters.

\section{Steady injection conditions}

The PIV data gathered in the axial plane and a transverse plane $2 \mathrm{~mm}$ above the top cone of the central bluffbody (flames $S_{0}$-bb, $S_{2}$-bb) and $2 \mathrm{~mm}$ above the injector outlet (flame $S_{2}$-as) are first used to determine the swirl number $S$ [4] at the injector outlet: $S=0.20$ for $S_{0}$-bb, $\mathrm{S}=0.80$ for $S_{2}$-bb and $\mathrm{S}=0.75$ for $S_{2}$-as, with a relative precision $\pm 3 \%$.

The swirl level for flame $S_{0}$-bb slightly differs from zero due to small imperfections in the swirler manufacturing. Several PIV measurements were made to check this feature that was found to be reproducible, with the same velocity profile, from tests to tests by mounting and demounting the swirler and the rod. Nonetheless, the swirl number $S=0.2$ remains in this case small and the configuration $S_{0}$-bb will be referred in the following as a non-swirling flame.

Effects of the swirl number $S$ on the shape taken by the flames are shown in Fig. 1 . The flame $S_{0}$-bb is an- 


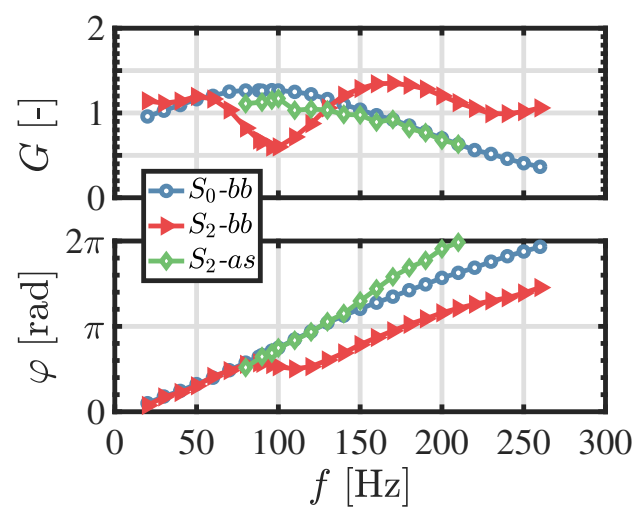

Fig. 2. Gain $G$ and phase lag $\varphi$ of the FTF for the three configurations investigated at the forcing level $u^{\prime} / \bar{u}=0.30$ RMS.

chored on the bluff body at the top in Fig. 1 with a relatively narrow reaction layer spreading over a long distance in the wake of the central bluff-body. The flame $S_{2}$-bb produced by the same injector but with a higher swirl $S=0.8$ is more compact in Fig. 1 . When the central rod is removed, Fig. 1 shows that the lifted flame $S_{2}$-as has about the same axial extent as flame $S_{0}$-bb but with an emission intensity peaking in the central region.

\section{Flame transfer functions}

The FTF of the three preceding flames is determined from the velocity signal measured by the hot wire anemometer and the $\mathrm{OH}^{*}$ chemiluminescence intensity $I$ measured by the photomultiplier gathering light from the whole combustion region. This signal is assumed to be a good tracer of the heat release rate. Flames are excited by the loudspeaker in the frequency range $20-260 \mathrm{~Hz}$ with a constant modulation level $u^{\prime} / \bar{u}=0.3$, where $\bar{u}$ and $u^{\prime}$ denote the mean and root-mean-square (RMS) values of the velocity signal measured by the hot wire. The FTF is deduced from the cross- and powerspectral densities of the hot wire and photomultiplier signals evaluated at the forcing frequency $f$ :

$$
\mathcal{F}(f)=\frac{I^{\prime} / \bar{I}}{u^{\prime} / \bar{u}}=G(f) \exp (i \varphi(f))
$$

Results are plotted in Fig. 2. The FTF gain curve of flame $S_{0}$-bb shows a typical low-pass filter behavior with a large gain overshoot at low frequencies, a well known feature of the acoustic response of laminar and turbulent premixed non-swirling V-flames anchored on a central bluff body $[17,18,26]$.

The FTF of the flame $S_{2}$-bb obtained with the same injector design, but a swirl level $S=0.80$, differs from flame $S_{0}$-bb by several aspects in Fig. 2. The FTF gain of $S_{2}$-bb shows a succession of a peak, a valley at $f_{0}=96 \mathrm{~Hz}$ and another peak over a short frequency range, over which the non-swirling flame $S_{0}$-bb response remains maximum. These features are now well understood and documented in a series of theoretical, numerical and experimental studies [7, 22, 23, 27]. They result from interferences between axial and azimuthal velocity fluctuations that are produced at the swirler outlet. It has been demonstrated that the frequency $f_{0}$ at which the FTF gain of a swirling flame reaches its minimum is obtained for the largest swirl number oscillation, i.e. a situation where azimuthal and axial velocity fluctuations are out of phase at the burner outlet [23].

The frequency $f_{0}$ can be roughly estimated by considering the phase-lag $\varphi_{u_{\theta}^{\prime}-u_{z}^{\prime}}$ between azimuthal and axial velocity fluctuations at the injector outlet [27, 28]:

$$
\varphi_{u_{\theta}^{\prime}-u_{z}^{\prime}}=2 \pi f_{0} \delta\left(\frac{1}{u_{c}}-\frac{1}{c}\right)
$$

where $u_{c}$ is the convection velocity of azimuthal disturbances and $c \gg u_{c}$ is the speed of sound. The condition leading to the highest swirl number oscillation amplitude, i.e. a minimum FTF gain, corresponds to $\varphi_{u_{\theta}^{\prime}-u_{z}^{\prime}}=\pi$. The distance $\delta$ in Eq. (2) is between the top of the swirler injection channels, where azimuthal disturbances are generated, and the combustor backplane. It is thus slightly smaller than the distance $L=56 \mathrm{~mm}$ between the swirler and the combustor backplanes. It was shown in $[11,23,28]$ that the velocity $u_{c}$ is close to the maximum velocity reached by the flow in the injection channel. This velocity remains unknown in the present study, but a rough estimate is made here by taking it equal to the highest axial velocity $u_{c}=9 \mathrm{~m} / \mathrm{s}$ measured at the injector outlet with PIV (not shown). The same choice was made in [23]. One finds $f_{0}=90 \mathrm{~Hz}$ for $u_{c}=9 \mathrm{~m} / \mathrm{s}$ and $\delta=50 \mathrm{~mm}$. This estimate is close to $f_{0}=96 \mathrm{~Hz}$ determined experimentally in Fig. 2 for flame $S_{2}$-bb. The largest uncertainty comes from the value of the convection velocity $u_{c}$, which depends on the flow structure between the swirler injection channel outlets and the burner outlet.

The FTF of the swirling flame $S_{2}$-as obtained without central insert does not feature any significant FTF gain drop in Fig. 2 even though the swirl level $S=0.75$ is close to the value $S=0.80$ for flame $S_{2}$-bb anchored on the bluff body. One may again roughly estimate the frequency $f_{0}$ associated to the largest swirl number oscillations. Considering the reduction of the injection tube diameter, one now takes $u_{c, 1}=5.44 \mathrm{~m} / \mathrm{s}$ over $\delta_{1}=16 \mathrm{~mm}$ and $u_{c, 2}=18.2 \mathrm{~m} / \mathrm{s}$ over $\delta_{2}=34 \mathrm{~mm}$ deduced from 
mass balance (see Fig. 1) and finds $f_{0}=104 \mathrm{~Hz}$. This frequency is well inside the range investigated but, nonetheless, there is no drop of the FTF gain in Fig. 2 for flame $S_{2}$-as.

Other interesting features, which seem to have not been reported previously, are observed for the FTF phase lag in Fig. 2. The phase lag curve of the nonswirling $S_{0}$-bb and highly swirled $S_{2}$-bb flames anchored on the central bluff body are the same at low frequencies $f<f_{0}$. This is not an obvious feature since the time lag $\tau$ appearing in the FTF phase lag $\varphi=2 \pi f \tau$ is generally proportional to the flame length $L_{f}$ and to the inverse of the bulk flow velocity $1 / U_{b}$. In Fig. 1, obtained for steady injection conditions, the flame length $L_{f}$ and flame aspect ratio largely differ between $S_{0}$-bb and $S_{2}$-bb while the bulk flow velocity remains the same. At higher frequencies $f>f_{0}$, the FTF phase lag of the non-swirling flame $S_{0}$-bb keeps increasing with an almost constant slope in the full frequency range investigated. An inflection point and a rapid change of the phase lag characterize the response of the highly swirled flame $S_{2}$-bb at frequencies close to $f \sim f_{0}$. At higher forcing frequencies, the data for the FTF phase lag of flame $S_{2}$-bb are found to be parallel to the FTF phase lag plot of the non-swirling flame $S_{0}$-bb offset by a constant value of $\varphi_{0} \simeq-1.3 \mathrm{rad}$. The FTF phase lag of the aerodynamically stabilized swirled flame $S_{2}$-as regularly increases with a different slope than flame $S_{0}$-bb and does not exhibit any inflection point.

\section{Flame dynamics}

Phase averaged images of the $\mathrm{OH}^{*}$ chemiluminescence conditioned by the harmonic excitation are examined in Fig. 3 to elucidate some of the previous observations. The forcing level $u^{\prime} / \bar{u}=0.30 \mathrm{RMS}$ is the same as in Fig. 2 showing the FTF results. The intensified CCD camera is synchronized with the signal driving the loudspeaker at the bottom of the burner. Images are taken for each configuration at the same phases separated by a constant interval of $30^{\circ}$. The phase angles are indicated in Fig. 3 with respect to the hot-wire signal below the radial swirler and a phase shift arises between flames forced at $96 \mathrm{~Hz}$ and $170 \mathrm{~Hz}$. An Abel deconvolution reveals the trace of the flame luminosity in an axial plane crossing the burner axis for flames $S_{0}$-bb and $S_{2}$-bb. This post-processing was not possible for flame $S_{2}$-as due to the too high intensity values close to the symmetry axis (see Fig. 1). The same color scale is used for all images to better highlight both the flame motion and changes of the flame luminosity during the forcing cycle.

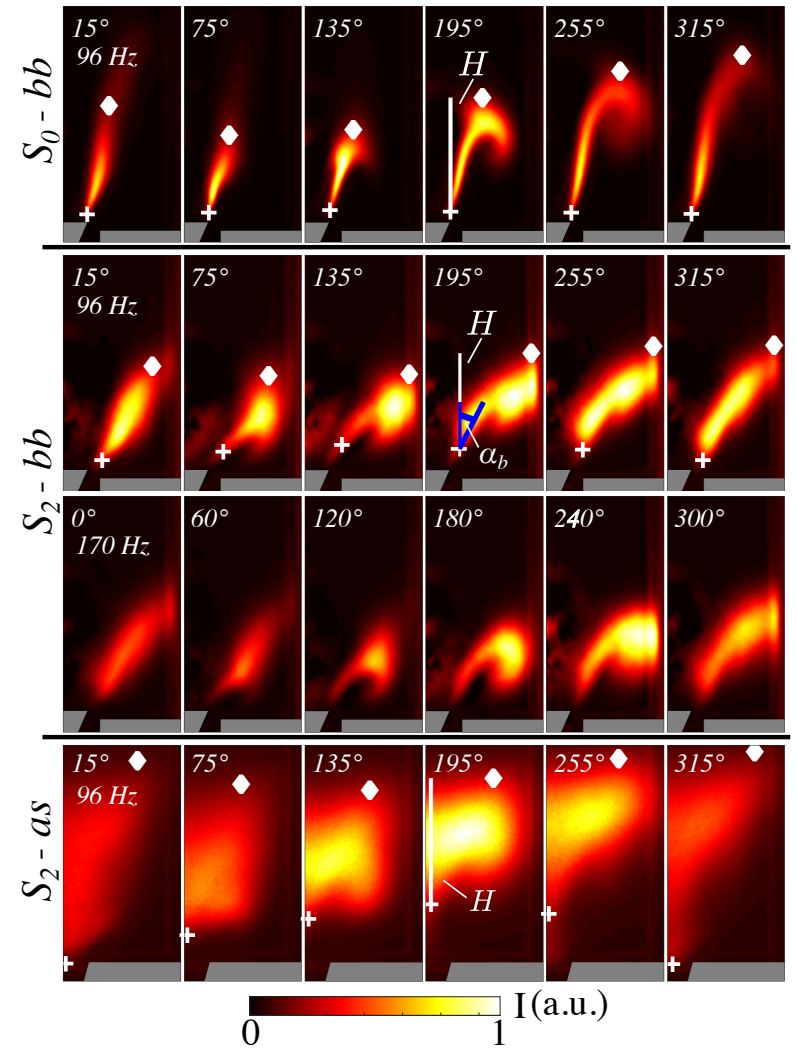

Fig. 3. $\mathrm{OH}^{*}$ intensity phase averaged images at a forcing level $u^{\prime} / \bar{u}=0.30$ RMS. + : flame root position. $\diamond$ : flame tip position.

The first sequence in Fig. 3 highlights the large motion undergone by the non-swirling flame $S_{0}$-bb at the forcing frequency $f=96 \mathrm{~Hz}$ when the FTF gain is maximum in Fig. 2. Large roll-up of the flame tip is seen at $195^{\circ}$ and $255^{\circ}$. The flame is stretched in the vertical direction during the forcing cycle with relatively minor changes of the $\mathrm{OH}^{*}$ luminosity.

The second and third sequences in Fig. 3 show the responses of flame $S_{2}-\mathrm{bb}$, at the FTF gain minimum at $f_{0}=96 \mathrm{~Hz}$ and at the FTF gain maximum at $f=170 \mathrm{~Hz}$ in Fig. 2. The motion undergone by the flame does not differ significantly at these two forcing frequencies, but it is mainly changes of the flame luminosity that explain the large differences observed for the FTF gain at $f_{0}=96 \mathrm{~Hz}$ and $f=170 \mathrm{~Hz}$ in Fig. 2. At $f_{0}=96 \mathrm{~Hz}$, there is a relatively weak flame roll-up motion accompanied by weak changes of the flame luminosity over the forcing cycle in Fig. 3. At $f=170 \mathrm{~Hz}$, the flame roll-up process is a bit further pronounced, but the $\mathrm{OH}^{*}$ luminosity undergoes large changes during the forcing cycle explaining the high value taken by the FTF gain at 

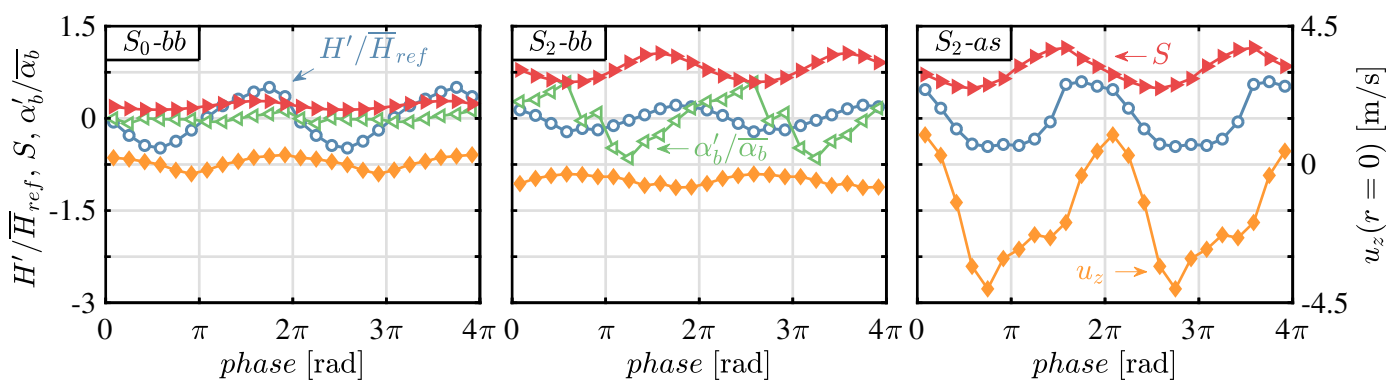

Fig. 4. Evolutions of the swirl number $\mathrm{S}$ and axial velocity $u_{z}(r=0)$ at the burner outlet with respect to the phase of the hot-wire signal. The relative flame height $H^{\prime} / \bar{H}_{r e f}$ and flame base angle $\alpha_{b}^{\prime} / \overline{\alpha_{b}}$ oscillations are also plotted. Two oscillation cycles are represented for better readability.

this frequency in Fig. 2.

The last sequence in Fig. 3 shows the dynamics of the aerodynamically stabilized flame $S_{2}$-as at $f=96 \mathrm{~Hz}$ corresponding to its peak FTF gain value in Fig. 2. The flame is rolled-up by vortex interaction $\left(75^{\circ}-195^{\circ}\right)$ and is stretched in the vertical direction, but also undergoes large changes of its luminosity as flame $S_{2}$-bb at $f=170 \mathrm{~Hz}$. The position of the leading edge of flame $S_{2}$-as also exhibits a large vertical oscillation during the forcing cycle, as highlighted by the white crosses in this sequence, while flames $S_{0}$-bb and $S_{2}$-bb remain anchored on the bluff body in Fig. 3 .

Further analysis is made at the forcing frequency $f_{0}=96 \mathrm{~Hz}$ by determining the average flame position for each image sequences. This profile is obtained by finding the maximum row-wise intensity of the pixel luminosity, weighted by the distance from the burner axis as expressed by: $\int I(r, x) 2 \pi r d r$. A threshold of $25 \%$ is selected to delineate the lower (cross symbol) and upper (diamond symbol) flame boundaries. For flame $S_{2}$-as in the last sequence in Fig. 3, the flame contour is used in place of the average flame position for the analysis.

This post-processing is used to deduce the flame height $H$ corresponding to the vertical distance between the upper (diamond sign) and lower (plus sign) flame boundaries i.e. the length of the vertical white segment shown in Fig. 3 at the phase $195^{\circ}$. This process proves to be efficient and robust even when the flame is strongly modulated by vortex interaction. The flame angle $\alpha_{b}$ with respect to the vertical direction is also determined at the flame leading edge position as shown in the second sequence in Fig. 3 at $195^{\circ}$. Evolutions of $H$ and $\alpha_{b}$ are plotted in Fig. 4 over two periods for the three flames $S_{0}$-bb, $S_{2}$-bb and $S_{2}$-as excited at $f=96 \mathrm{~Hz}$. The same reference height $\bar{H}_{\text {ref }}=25 \mathrm{~mm}$ is used to normalize the results. The three flames exhibit a modulation of their height $H$ during the forcing cycle, but the oscillation amplitude $H^{\prime}$ is a bit lower for $S_{2}$-bb compared to
$S_{0}$-bb and $S_{2}$-as. The swirling flame $S_{2}$-bb stabilized by the bluff body also features a large oscillation of the flame angle $\alpha_{b}$ at its base at $f_{0}=96 \mathrm{~Hz}$. These flame angle oscillations are not observed for the non-swirling flame $S_{0}$-bb and could not be determined for the fully aerodynamically stabilized flame $S_{2}$-as.

Figure 4 also shows the evolution of the swirl number $S$ at the injector outlet, determined, for each selected phase in the cycle, by PIV measurements conducted in cold flow conditions, with a relative precision of $\pm 5 \%$. One clearly identifies a large modulation of the swirl level at $f=96 \mathrm{~Hz}$ for the swirling flames $S_{2}$-bb with and $S_{2}$-as without bluff body, while at the same frequency there are no swirl oscillations for the flame $S_{0}$-bb. This analysis confirms that both flames, $S_{2}$-bb and $S_{2}$-as, undergo large swirl number oscillations at $f=96 \mathrm{~Hz}$, but their FTF largely differ even though they share about the same swirl level $S \sim 0.8$.

Finally, the axial velocity signal $u_{z}$ on the symmetry axis $r=0$ is examined in Fig. 4. This signal measured $2 \mathrm{~mm}$ above the top cone is barely altered by the flow modulation at $f_{0}=96 \mathrm{~Hz}$ for flames $S_{0}$-bb and $S_{2}$-bb anchored in the wake of the central bluff body. This contrasts with the large oscillation in Fig. 4 observed for the same signal measured $2 \mathrm{~mm}$ above the injector outlet for the swirling flame $S_{2}$-as without bluff body. This large modulation is responsible for the displacement of the leading edge position of the aerodynamically stabilized flame $S_{2}$-as in the bottom image sequences in Fig. 3 .

Further analysis is now carried out under cold flow conditions by examining the dynamics of coherent vortical disturbances synchronized by the acoustic pulsation. Results are presented in Fig. 5. To identify vortical structures, the Q criterion [29] is selected:

$$
Q=\frac{1}{2}\left(|\mathbf{\Omega}|^{2}-|\mathbf{S}|^{2}\right)
$$

where $\mathbf{S}$ and $\boldsymbol{\Omega}$ are the symmetric and anti-symmetric 


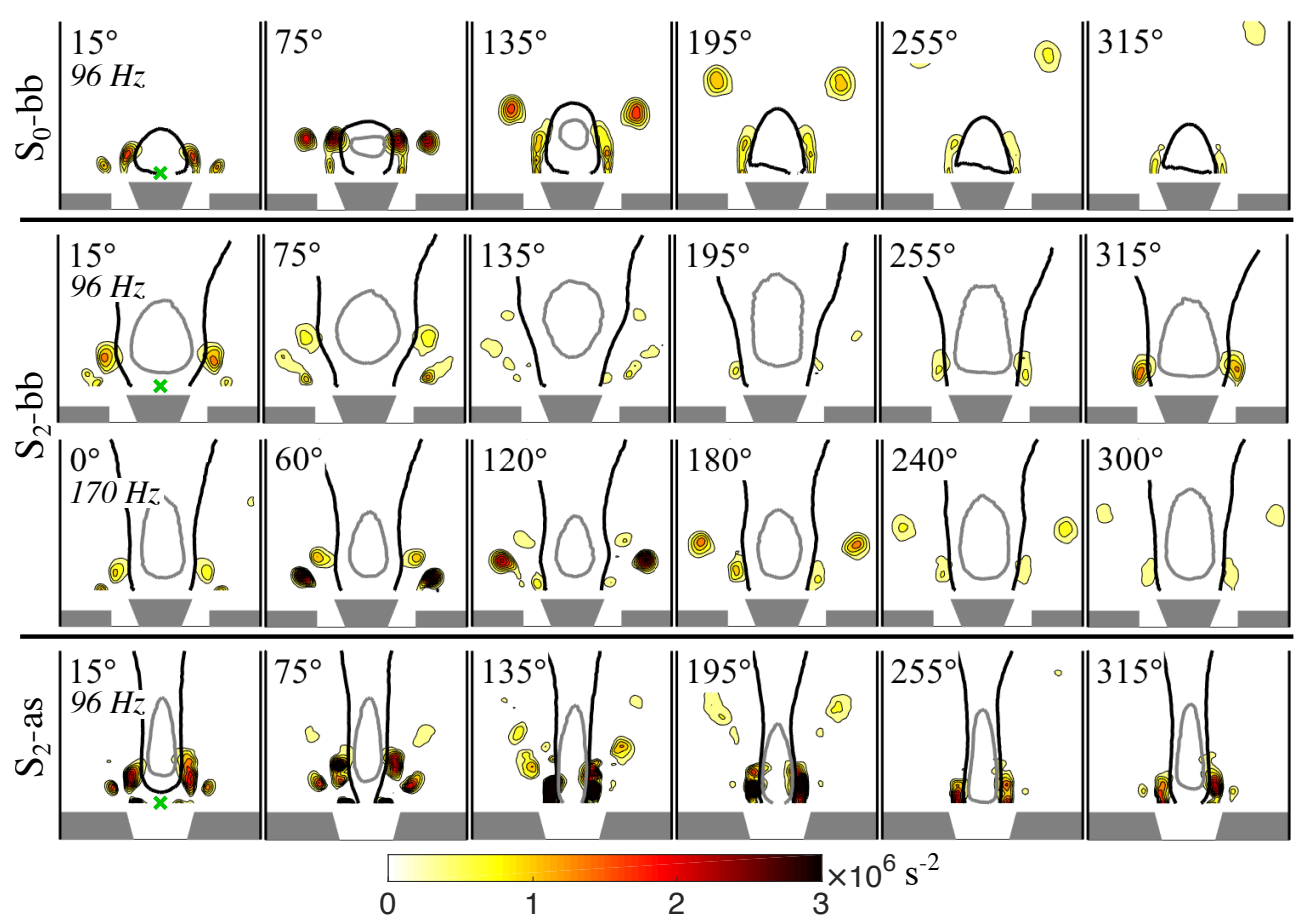

Fig. 5. Q criterion contour obtained from PIV measurements in cold flow conditions, for a forcing level $u^{\prime} / \bar{u}=0.30$ RMS. Isolines of axial velocity are superimposed. Black contour: $u_{z}=0 \mathrm{~m} / \mathrm{s}$. Gray contour: $u_{z}=-2.5 \mathrm{~m} / \mathrm{s}$. The position where the axial velocity $u_{z}$ for the analysis of Fig. 4 is measured, is shown as a green cross at the phase $15^{\circ}$.

components of the velocity gradient respectively. Isocontours of $Q$ are inferred from PIV measurements in the axial plane. Only positive values of $Q$ are retained. Negative values, indicating regions where shear is present but no swirling motion, are forced to a zero value.

In the first, third and last sequences in Fig. 5, corresponding to FTF gain maxima in Fig. 2, large vortical structures generated at the rim of the injector are produced by the acoustic forcing and convected downstream in the chamber. In the second sequence in Fig. 5 corresponding to the FTF gain minimum in Fig. 2 for flame $S_{2}$-bb at $f_{0}=96 \mathrm{~Hz}$, vortical structures are much weaker as emphasized by the much lower values taken by the $\mathrm{Q}$ criterion. As a consequence, the flame response remains low at this forcing frequency.

Isolines of axial velocity are also shown in Fig. 5, to highlight the different dynamics of the internal recirculation zone depending upon the flame stabilization mechanism. Flame $S_{0}$-bb feature a small recirculation region in the wake of the bluff-body. Flame $S_{2}$-bb feature a larger IRZ, undergoing a flapping motion during the forcing cycle, which is more evident at $f_{0}=96 \mathrm{~Hz}$. When the flame is stabilized without bluff-body, $S_{2}$-as, the IRZ is much thinner and oscillates vertically in and out of the injector.

These observations are confronted with current interpretations of the response of swirling flames associated with the combined effects of swirl number oscillations and flame vortex roll-up. Palies et al. [19] explained that at the FTF gain minimum, large swirl number oscillations modulate the strength of the IRZ. This oscillation weakens the formation of eddies and also leads to flame base angle oscillations. When the flame angle fluctuates, vortex growth is rapidly hindered by the flame flapping motion and the flame response is low. When the flame angle oscillation weakens, the response is high because vortices can fully develop before interacting with the flame. Interaction of vortex shedding and flame angle fluctuations has been further analyzed in [20]. These authors argue that as the flame moves closer to the shear layer, the vorticity of the flow is dissipated due to its interaction with the flame and the flame response is low. In contrast, when the mean flame position is stationary, the vorticity of the flow is not dissipated before interacting with the flame, which leads to a large flame response.

The observations made in this work are for some aspects consistent with these conclusions, but also reveal new mechanisms. Figure 4 confirms that swirl num- 
ber and flame base angle oscillations are large for flame $S_{2}$-bb stabilized by a bluff body at $f_{0}=96 \mathrm{~Hz}$ where the FTF gain is at a minimum. It is however found that the formation of large vortical structures are damped even without combustion. This phenomenon is here not related to the flapping motion of the flame, but is found to be related to the geometry of the injector. At the same excitation frequency, large vortical structures are shed from the injector without bluff-body, while large swirl level oscillations are also observed. In this case, the response of flame $S_{2}$-as remains high. This flame also exhibits a large vertical oscillation of its leading edge and a peak value of the FTF gain at $f=96 \mathrm{~Hz}$. This analysis reveals that the mechanisms controlling the frequency response of swirled flames largely differ when they are stabilized by a bluff body or when they are stabilized aerodynamically, the main differences being related to the dynamics of the IRZ.

\section{Conclusion}

Transfer functions of flames stabilized with different injector designs have been investigated for different swirl levels, with and without a central insert in the injector. Depending on the stabilization mechanism, flame vortex roll-up, oscillations of the flame base angle induced by swirl level oscillations and vertical oscillations of the flame leading edge, are found to be the competing mechanisms controlling the flame response. When the FTF gain is at a maximum, the three flames investigated are strongly modulated by the interaction with large vortical structures convected in the external shear layer of the flow, regardless of changes of the swirl level and stabilization mechanisms. In contrast, the formation of large vortical structures is hindered, even in cold flow conditions, at the frequency corresponding to a minimum in the FTF gain curve, which is observed in the present study only for the swirling flame stabilized by a bluff body. At this frequency, large swirl number oscillations lead to a modulation of the flame base angle and weak vortex formation. At the same frequency, but for the aerodynamically stabilized flame without central insert, swirl number oscillations of the same order of magnitude are observed, but the gain of the FTF remains high. In this case, large vortical structures are shed and the flame leading edge oscillates vertically. The origin of the low response of swirling flames at specific frequencies is found to not only be related to large oscillations of the swirl level, but also to the flame stabilization mechanism and more specifically to the dynamics of the internal recirculation region.

\section{Acknowledgements}

This project has received funding from the European Union's Horizon 2020 research and innovation programme under the Marie Sklodowska-Curie grant agreement No 643134. This work is supported by Agence Nationale de la Recherche, NOISEDYN project (ANR-14-CE35-0025-01).

\section{References}

[1] T. C. Lieuwen, V. Yang, Combustion Instabilities: Basic Concepts, 2005.

[2] Y. Huang, V. Yang, Prog. Energy Combust. Sci. 35 (2009) 293364.

[3] T. Poinsot, Proc. Combust. Inst. 36 (2017) 1-28.

[4] S. Candel, D. Durox, T. Schuller, J.-F. Bourgouin, J. P. Moeck, Annu. Rev. Fluid Mech. 46 (2014) 147-173.

[5] S. K. Thumuluru, T. Lieuwen, Proc. Combust. Inst. 32 (2009) 2893-2900.

[6] C. Hirsch, D. Fanaca, P. Reddy, W. Polifke, T. Sattelmayer, in: Proc. ASME Turbo Expo 2005, GT2005-68195.

[7] P. Palies, T. Schuller, D. Durox, S. Candel, Proc. Combust. Inst. 33 (2011) 2967-2974.

[8] V. Acharya, Shreekrishna, D.-H. Shin, T. Lieuwen, Combust. Flame 159 (2012) 1139-1150.

[9] L. Tay Wo Chong, R. Kaess, T. Komarek, S. Foller, W. Polifke, in: Proc. ASME Turbo Expo 2010, GT2010-22769.

[10] F. Biagioli, B. Paikert, F. Genin, N. Noiray, S. Bernero, K. Syed, Flow, Turbul. Combust. 90 (2013) 343-372.

[11] V. Acharya, T. Lieuwen, Phys. Fluids 27 (2015) 105106.

[12] C. Kulsheimer, H. Buchner, Combust. Flame 131 (2002) 70-84.

[13] D. Kim, J. G. Lee, B. D. Quay, D. A. Santavicca, K. Kim, J. Eng. Gas Turbines Power 132 (2010) 757-765.

[14] P. Palies, D. Durox, T. Schuller, S. Candel, Combust. Sci. Technol. 183 (2011) 704-717.

[15] B. Ćosić, S. Terhaar, J. P. Moeck, C. O. Paschereit, Combust. Flame 162 (2015) 1046-1062.

[16] B. Schuermans, F. Guethe, D. Pennell, D. Guyot, C. O. Paschereit, J. Eng. Gas Turbines Power 132 (2010) 111503.

[17] D. Durox, T. Schuller, S. Candel, Proc. Combust. Inst. 30 (2005) 1717-1724.

[18] R. Balachandran, B. O. Ayoola, C. F. Kaminski, A. P. Dowling, E. Mastorakos, Combust. Flame 143 (2005) 37-55.

[19] P. Palies, T. Schuller, D. Durox, L. Y. Gicquel, S. Candel, Phys. Fluids 23 (2011) 037101.

[20] N. A. Bunce, B. D. Quay, D. A. Santavicca, J. Eng. Gas Turbines Power 136 (2013) 021503.

[21] K. Oberleithner, S. Schimek, C. O. Paschereit, Combust. Flame 162 (2015) 86-99.

[22] T. Komarek, W. Polifke, J. Eng. Gas Turbines Power 132 (2010).

[23] P. Palies, D. Durox, T. Schuller, S. Candel, Combust. Flame 157 (2010) 1698-1717.

[24] R. Gaudron, M. Gatti, C. Mirat, T. Schuller, in: Proc. ASME Turbo Expo 2018, GT2018-76381.

[25] F. Giuliani, P. Gajan, O. Diers, M. Ledoux, Proc. Combust. Inst. 29 (2002) 91-98.

[26] T. Schuller, D. Durox, S. Candel, Combust. Flame 134 (2003) 21-34.

[27] K. T. Kim, D. A. Santavicca, Combust. Flame 160 (2013) 14411457.

[28] P. Palies, D. Durox, T. Schuller, S. Candel, J. Fluid Mech. 672 (2011) 545-569.

[29] J. Jeong, F. Hussain, J. Fluid Mech. 285 (1995) 69-94 